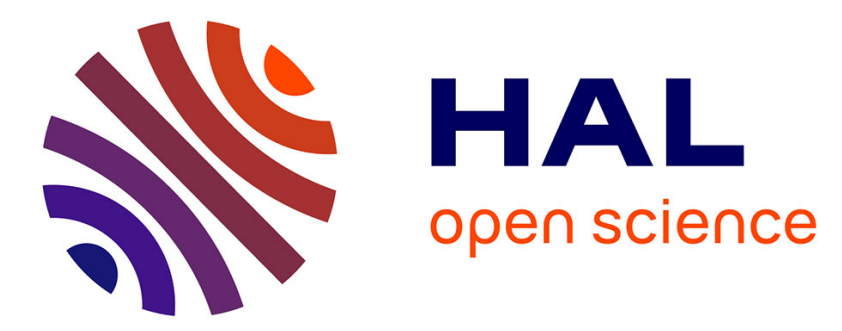

\title{
EMG artifacts removal during electrical stimulation, a CWT based technique
}

\author{
Maxime Yochum, Toufik Bakir, Stéphane Binczak, Romuald Lepers
}

\section{To cite this version:}

Maxime Yochum, Toufik Bakir, Stéphane Binczak, Romuald Lepers. EMG artifacts removal during electrical stimulation, a CWT based technique. IEEE Region 10 Symposium, Apr 2014, Kuala Lumpur, Malaysia. 10.1109/TENCONSpring.2014.6863012 . hal-01328483

\section{HAL Id: hal-01328483 \\ https://hal.science/hal-01328483}

Submitted on 8 Jun 2016

HAL is a multi-disciplinary open access archive for the deposit and dissemination of scientific research documents, whether they are published or not. The documents may come from teaching and research institutions in France or abroad, or from public or private research centers.
L'archive ouverte pluridisciplinaire HAL, est destinée au dépôt et à la diffusion de documents scientifiques de niveau recherche, publiés ou non, émanant des établissements d'enseignement et de recherche français ou étrangers, des laboratoires publics ou privés. 


\title{
EMG artifacts removal during electrical stimulation, a CWT based technique
}

\author{
Maxime Yochum, Toufik Bakir, Stéphane Binczak \\ LE2I UMR CNRS 6306 \\ University of Burgundy \\ 9 avenue Alain Savary \\ 21078 Dijon, France \\ stbinc@u-bourgogne.fr
}

\author{
Romuald Lepers \\ Laboratory INSERM U1093 \\ University of Burgundy \\ Campus Universitaire \\ 21078 Dijon, France \\ romuald.lepers@u-bourgogne.fr
}

\begin{abstract}
A technique of artifacts removal based on the continuous wavelet transform is presented. It uses common mother wavelets to find the temporal localization of stimulation artifacts on electromyogram (EMG) signal during an electrically evoked contraction of a muscle. This method can be used with standard stimulation pulse waveforms like monophasics or biphasics ones. It uses a histogram representation to find the best threshold to apply on the CWT domain. The algotithm is presented with Haar wavelet and then it is used with common wavelet famillies such as Daubechies or Symlets.
\end{abstract}

\section{Keywords}

Electromyogram, stimulation artifacts, source separation, wavelet, histogram representation.

\section{INTRODUCTION}

Electromyogram (EMG) corresponds to the electrical response of the muscle over its contraction. During a myo-electrical stimulation, EMG contains two sources. The first one is the muscular electrical activity generated by the stimulated muscle due to the contraction of its muscular fibers. This signal is named $\mathrm{M}$ wave signal and it is a useful signal to study physiological states of muscles such as force or fatigue. The second one is the artifacts corresponding to the electrical stimulation pulses contained in EMG. Those artifacts disturb the analysis of electrical muscular activity. In order to analyze properly the EMG signal, artifacts need to be removed [1]. Several techniques are already used to attenuate the impact of artifacts on EMG signals. Some of them are implemented on the hardware. They are placed just after the EMG electrodes in the differential amplifier circuitry. For instance, analog filtering where high frequencies of EMG, which contain artifacts, are removed. It is very difficult to filter artifacts without modifying M waves. For instance, a 8th order Chebyshev low pass filter with a $550 \mathrm{~Hz}$ cutoff frequency has been implemented by Solomonow et al. [2] to remove the most part of artifact frequencies. However, those kinds of filters let pass low frequencies artifact and could remove $\mathrm{M}$ waves frequencies which are in the same frequency range than artifacts. Their are also methods based on hardware amplifier gain. For instance, Roskar et al. [3] manage the amplifier gains to obtain a gain of 1 during the electrical stimulation and a gain of 1000 elsewhere. However, a gain of 1000 could not be sufficient to remove completely artifacts [1] and would be unable to adapt to electrical stimulation changes. Blanking hardware methods are also available, that consists to disconnect electrically EMG electrodes of muscle during electrical stimulation pulses. Generally, a synchronized triggering from the electrical stimulator is used [4], [5]. The drawback of this technique is that the blanking window is fixed and can lead to residual artifacts if the blanking time is too short or can lead to remove some $\mathrm{M}$ waves parts if the blanking time is too long. Other techniques are implemented in software with signal processing as a pre-processing of EMG signal. For instance, some methods use the recording of stimulation artifact signal in order to subtract it on EMG. Three main techniques exist to record an estimation of stimulation artifact signal: a stimulation below the muscular contraction threshold (stimulation does not cause any muscle contraction), a recording far away from the EMG electrodes placement (a second EMG is collected away from the first one to acquire pure artifacts), a dual pulse stimulation (a second stimulation pulse is done during the refractory period which does not create any evoked signal, only the stimulus artifact is collected) [6]. Those techniques do not remove entirely artifacts and tend to degrade the signal to noise ratio (SNR). In addition, they need a supplementary hardware part for the most of them. There are also methods based on threshold detection [7]. Stimulation artifacts are mostly higher than M wave amplitude with a factor of 2 or 3 [1]. This difference of amplitude leads to share the artifact and the M wave parts. Nevertheless, it may not be suitable to specific stimulations with small amplitude signals. Some other mathematical methods estimate stimulation artifacts in order to remove them [8].

In this study, we propose a new method to remove stimulation artifacts on EMG during an electrical stimulation rehabilitation process by using a continuous wavelet transform (CWT) and a histogram representation. It shows a bimodal distribution where artifacts and $\mathrm{M}$ waves can be shared. The histogram is then used to find the best threshold in the wavelet domain in order to correctly remove the artifacts from EMG. Haar mother wavelet is firstly used to present the removal method and then results 
with the use of some other mother wavelets are displayed to determine differences among wavelet families results. This artifacts removal algorithm can be used as threshold removal technique with automatical determination of the threshold which can be difficult to estimate when it is fixed such as in [7].

\section{MATERIAL}

The material of our experiments allows the electrical stimulation and the acquisition of muscular EMG in real time [9]. An electrical stimulator generates controlled current pulses and can deliver constant stimulation over time for different muscles. It can deliver a maximum of $\pm 100 \mathrm{~mA}$ for a body impedance of $1.5 \mathrm{~K} \Omega$ between the two stimulation electrodes, a frequency pulse train from $10 \mathrm{~Hz}$ to $100 \mathrm{~Hz}$ and a pulse time from $0.5 \mathrm{~ms}$ to $2 \mathrm{~ms}$ for 5 different pulse shapes which are the most common in literature (monophasic, biphasic, dual biphasic, biphasic amorted and N-let). An EMG amplifier acquires EMG from muscle during the stimulation with bipolar electrodes and a reference electrode. Amplifiers used for the circuit are INA128 from Texas instruments with a $120 d B$ of Common Mode Rejection Ratio (CMRR) for a good removal of common voltage on bipolar electrodes. The M waves can be extracted from global EMG signal during evoked contractions. M waves are then used to quantify the muscular fatigue level [10]. A software manages stimulation parameters and analyzes the fatigue indexes of a muscle after an artifacts pre-processing removal choosen among three methods: blanking, threshold algorithm or the method proposed in this study. Our experiments have been performed on the right biceps muscle isometrically thanks to a Biodex pro 3 device [11] on five subjects. Ten electrical stimulations of six seconds have been performed on each subject with different stimulation parameters which provided fifty recorded data for our experiments.

\section{Methods}

We propose a method based on wavelets to detect and remove the stimulation artifacts which are present in the EMG signal. This method uses the continuous wavelet transform to detect artifacts. The idea is to use a standard wavelet, as the Haar wavelet, and to vary the scale factor of the continuous wavelet transform to determine which scale factor corresponds to the maximum correlation between the stimulation artifact and the wavelet subjected to the scale factors. An example is provided in the figure 1 . Then a histogram representation is used to determine the best threshold in the wavelet domain to share the best artifacts and $\mathrm{M}$ waves. Nevertheless, the treatment should leave the $\mathrm{M}$ waves unchanged between stimulation artifacts to allow the extraction of physiological muscle states parameters. Initially, in order to explain the removal process, CWT was based on the Haar wavelet. Then, tests used other standard mother wavelets such as Daubechies or Symlets for instance.

\section{A. scale factor determination}

The Haar wavelet has the particularity to look like the stimulation artifacts in the sense that both have large edge changes. This wavelet should therefore properly detect artifacts. The continuous wavelet transform is applied between EMG with artifacts named $\mathrm{V}_{\mathrm{EMG}}$ and a wavelet $\psi$ (Haar wavelet) undergoing the scale factor $a$. Such as

$$
C_{a, b}\left(V_{E M G}(t), \psi(t)\right)=\int_{-\infty}^{\infty} V_{E M G}(t) \frac{1}{\sqrt{a}} \psi\left(\frac{t-b}{a}\right) d t
$$

where $b$ is the temporal parameter. The scale factors have been chosen to go from 1 to 100 with step of 1 . This range allows the analyzis of many different artifact durations. In the figure 1, a result of a continuous wavelet transform with the above parameters is shown. The used $\mathrm{V}_{\mathrm{EMG}}$ signal corresponds to that one in the figure $2 \mathrm{a}$. In order to find which scale factor $a$ corresponds to the maximum of correlation with artifacts of $\mathrm{V}_{\mathrm{EMG}}$, we need to estimate which scale factor corresponds to the maximal value of the CWT coefficients matrix, named $C_{a, b}$. In the figure 1 , an example of $C_{a, b}$ is plotted where the value of the best scale factor name $a_{0}$ is obtained with the maximum value of $C_{a, b}$ matrix.

\section{B. mask removal determination}

Once the $a_{0}$ scale factor has been found, we determine a temporal mask in the wavelet domain, allowing the removal of artifacts. To perform that, we use the CWT coefficients $C_{a, b}$ which correspond with this $a_{0}$ scale factor (it is then a coefficients vector $C_{a_{0}, b}$ ). In the figure $2 \mathrm{~b}$, an example of a continuous wavelet transform is shown on a $\mathrm{V}_{\mathrm{EMG}}$ signal recorded with an electrical stimulation at a $30 \mathrm{~Hz}$ frequency, a $40 \mathrm{~mA}$ amplitude, $1 \mathrm{~ms}$ pulse time duration and a biphasic waveform. Only the 0.2 first second of the 6 seconds stimulation are displayed for a better readability. The found $a_{0}$ scale factor was 38 . The curve in the graphic $\mathrm{b}$ corresponds to the continuous wavelet transform $C_{a, b}$ with Haar mother wavelet and the scale factor $a=a_{0}$. It is interesting to see that $C_{a_{0}, b}$ values corresponding to artifacts are much more important than $C_{a_{0}, b}$ values corresponding to $\mathrm{M}$ waves. Thanks to this point, it is possible to distinguish both artifacts and $\mathrm{M}$ waves parts. A threshold is created with local maxima algorithm which is used to find maxima coefficients during stimulation artifacts and maxima coefficients during $\mathrm{M}$ waves. In the figure $2 \mathrm{~b}$, a representation of this detection is shown with crosses (during artifacts) and plus (during $\mathrm{M}$ waves). In the figure $2 \mathrm{c}$, the histogram of these specific points is plotted and shows a bimodal distribution. The threshold which allows 


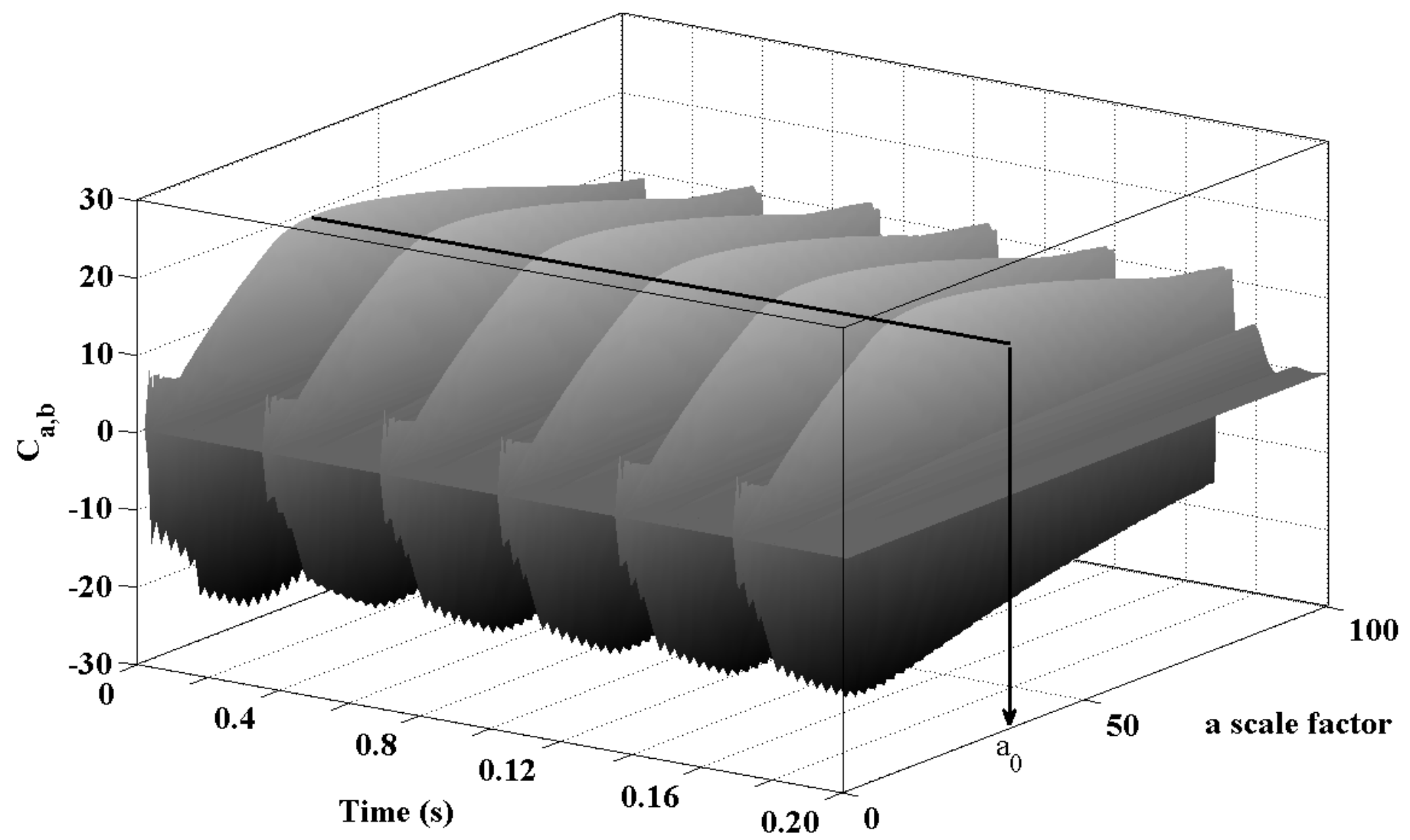

Fig. 1. Example of continuous wavelet transform result with Haar mother wavelet from $\mathrm{V}_{\mathrm{EMG}}$ signal plotted in the figure $2 \mathrm{a}$. scale factors go from 1 to 100 . The $a_{0}$ scale factor corresponding with the maximum of $C_{a, b}$ is represented with the black arrow line.

distinguishing stimulation artifacts without disturbed $\mathrm{M}$ waves is located between those two distributions. We use the centroid of these points in the histogram as our threshold because it shares correctly both distributions. The centroid is computed as:

$$
h=\frac{\sum_{i=1}^{n} x_{i} y_{i}}{\sum_{i=1}^{n} y_{i}},
$$

where $h$ is the centroid value, $x_{i}$ are abscissa of the histogram, $y_{i}$ are ordinates of the histogram and $n$ represents the histogram range. Then, when the threshold is computed, a mask removal is created by using $\left|C_{a_{0}, b}\right|$ absolute coefficients values and the threshold $h$. The mask is equal to 1 if $\left|C_{a_{0}, b}\right|$ are below the threshold, which corresponds to the $\mathrm{M}$ wave parts. The mask is equal to 0 if $\left|C_{a_{0}, b}\right|$ are above the threshold, corresponding to the stimulation artifacts parts. Therefore,

$$
\begin{cases}m_{p}=1 & \text { if }\left|C_{a_{0}, b}\right|<h, \\ m_{p}=0 & \text { if }\left|C_{a_{0}, b}\right| \geq h,\end{cases}
$$

where $m_{p}$ is the preliminary mask. In the figure 3 , an example of mask removal creation is shown. The $C_{a_{0}, b}$ coefficient values are plotted in the graphic a and the threshold is represented with the dash lines. We can note that during the stimulation artifacts (see Fig. $3 \mathrm{~b}$ ), the preliminary mask $m_{p}$ can change states. To correct that, an erosion algorithm (a mathematical morphology operation [12]) is applied to the preliminary mask vector and gives the final mask $m$. An example of the corrected mask after erosion is shown in the figure $3 \mathrm{c}$.

\section{Stimulation artifacts removal}

The last step of artifacts removal consists to multiply the mask created previously with $\mathrm{V}_{\mathrm{EMG}}$ signal to obtain the EMG signal without stimulation artifacts named $M_{\text {wave }}$.

$$
M_{\text {wave }}(t)=V_{E M G}(t) \cdot m(t)
$$



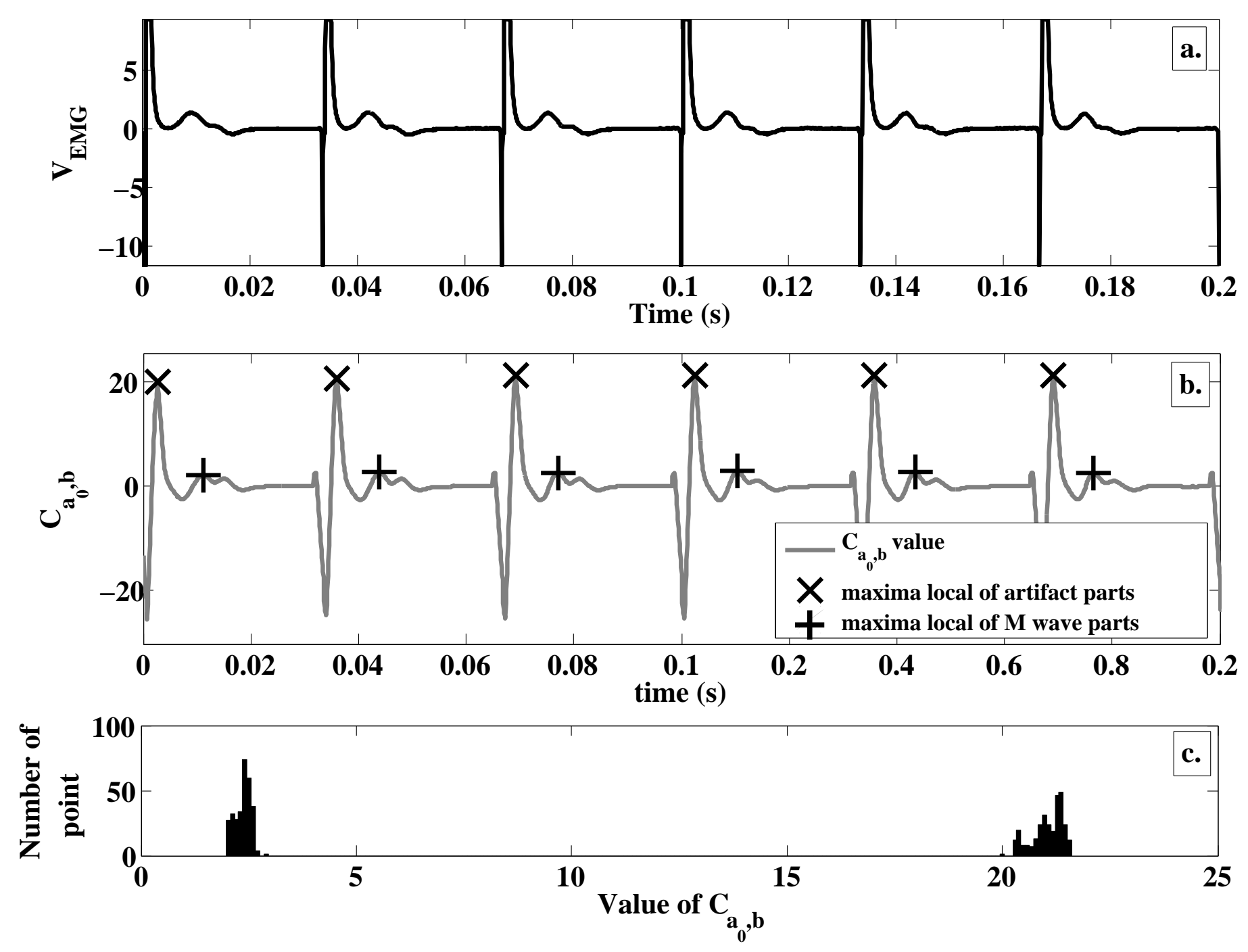

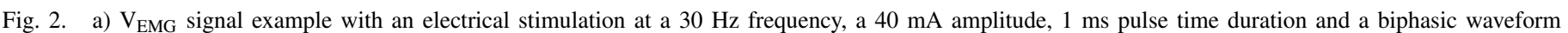

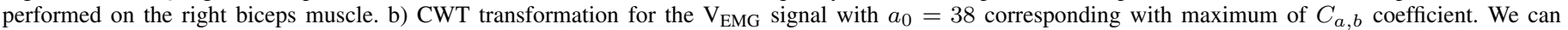

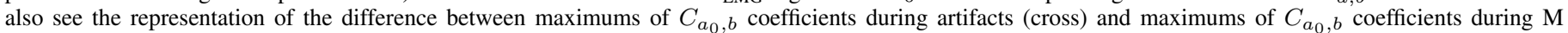
waves (plus) which allows the differentiation between artifacts and $\mathrm{M}$ waves. c) Histogram of maxima local values. We can discern a bimodal distribution.

An example of $M_{\text {wave }}$ is shown in the figure $4 \mathrm{~b}$ which corresponds to the stimulation artifacts removal performed on the $\mathrm{V}_{\mathrm{EMG}}$ signal with an electrical stimulation at $30 \mathrm{~Hz}$ frequency, $40 \mathrm{~mA}$ intensity, $1 \mathrm{~ms}$ pulse time duration and a biphasic waveform represented in the figure $4 \mathrm{a}$. As we can see, artifacts are completely removed from $\mathrm{V}_{\mathrm{EMG}}$ signal. Only $\mathrm{M}$ wave parts are preserved. The CWT with Haar mother wavelet is therefore a good domain to remove stimulation artifacts with our technique. As we can see, the $\mathrm{M}$ wave remains unchanged after the artifacts removal process.

\section{RESULTS}

The previous section explains the algorithm with Haar mother wavelet but it can also be used with others common mother wavelets. In the figure 5 and 6, we can see the use of other mother wavelets which are widely used in CWT treatments. Mother wavelets used are issued from Daubechies, Symlets, Coiflet, Gaussian, Morlet, Meyer, Biorthogonal, and Shannon wavelets. We can see that the majority of mother wavelets gives good results with a good removal of stimulation artifacts. However, some are less efficient than others. It is the case for "Sym3" and "shan1-1" which do not remove completely artifacts. If parts of artifacts remain in the $M_{\text {wave }}$ signal, they could lead to EMG estimation errors in future treatment. It is also the case for "gaus3" and "meyr". This time, the artifact parts are completely removed but the issue is that the M wave parts are truncated and could lead to $\mathrm{M}$ wave estimation errors in future treatment. The Haar wavelet gives good result (as shown in the figure 4) and it is also the easiest one to apply, therefore we recommend the use of this one with this algorithm. 


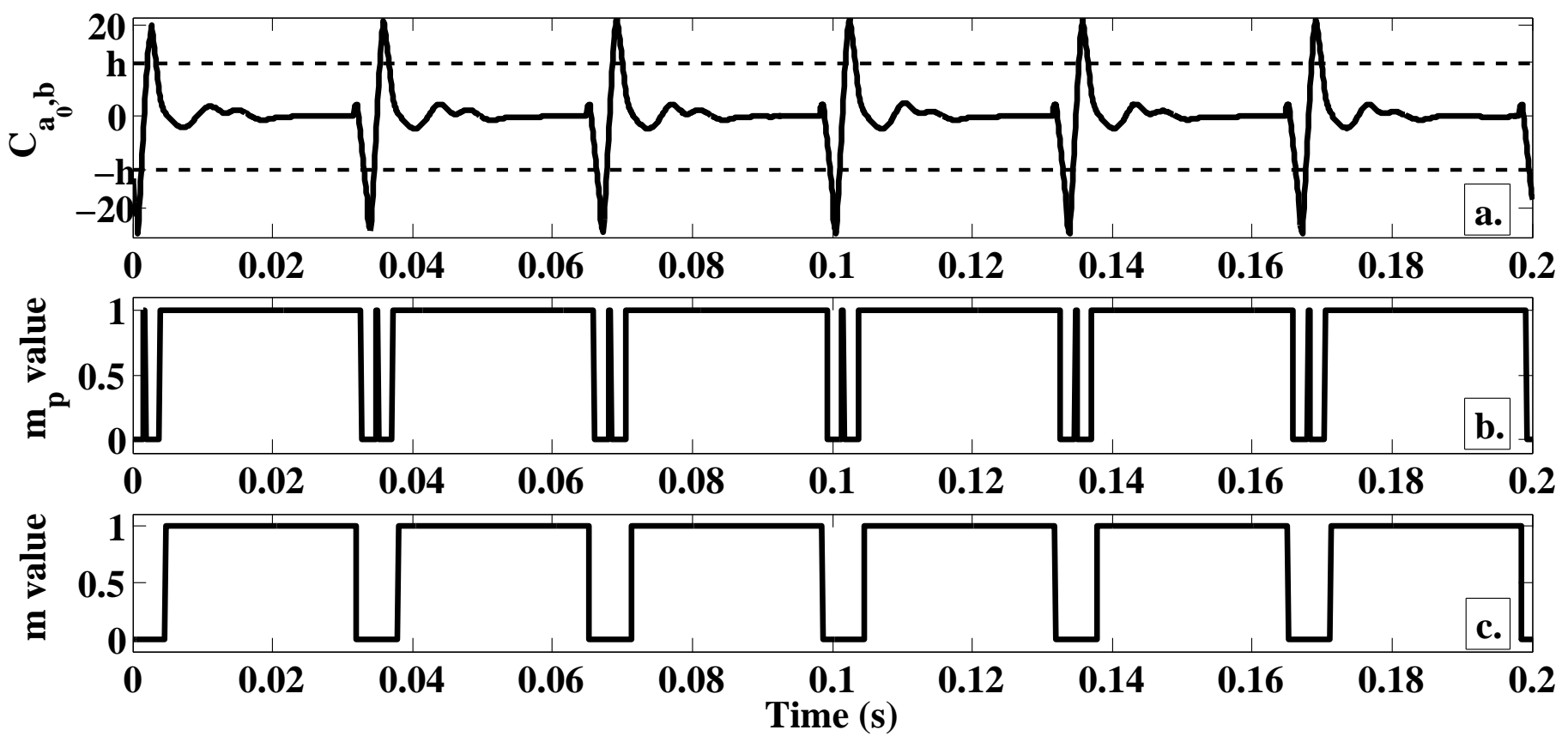

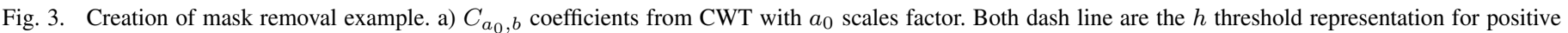
and negative parts computed with the equation 2. b). Preliminary $m_{p}$ mask created with $h$ threshold. c) Final $m$ mask from an erosion on $m_{p}$.
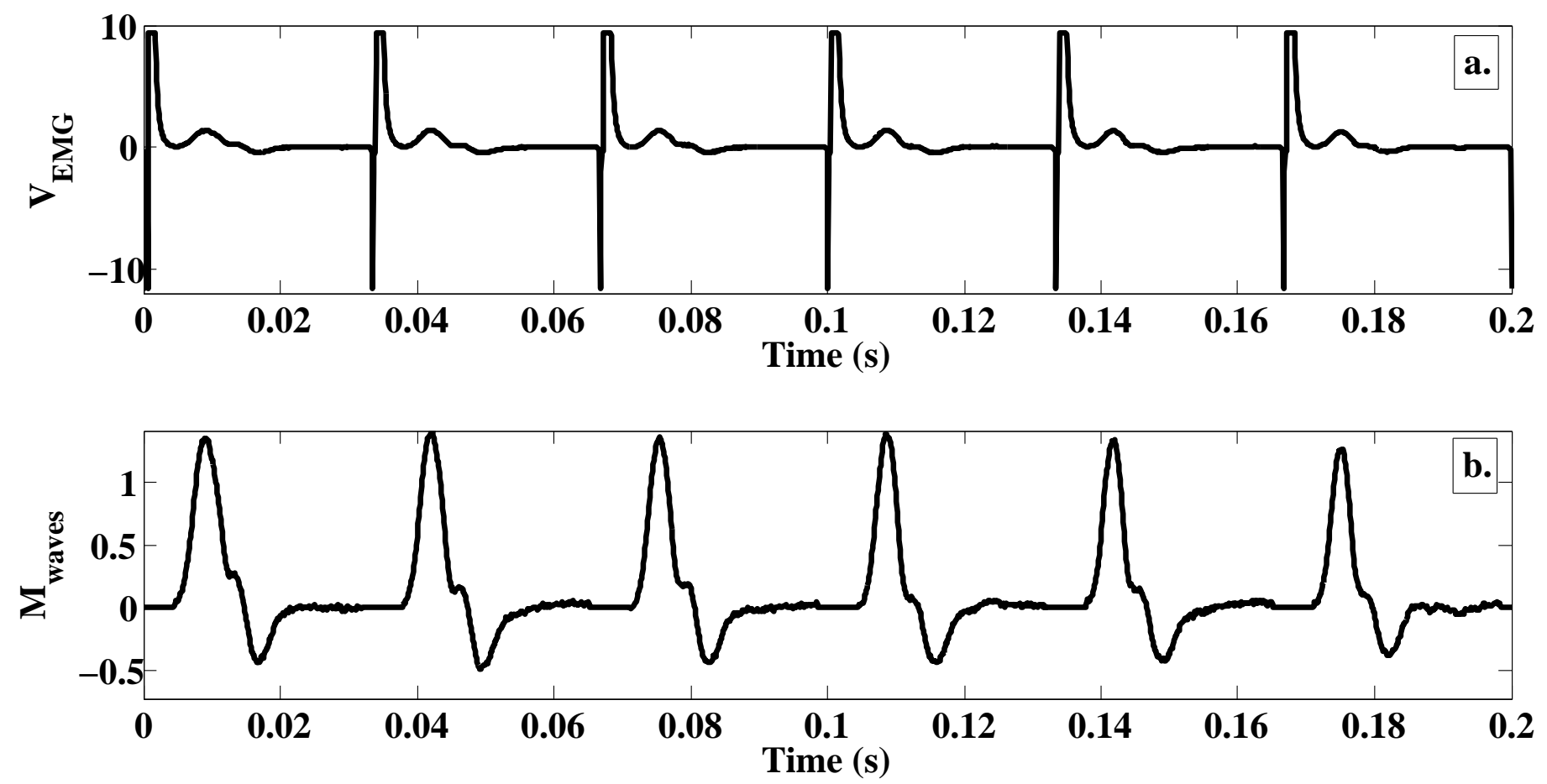

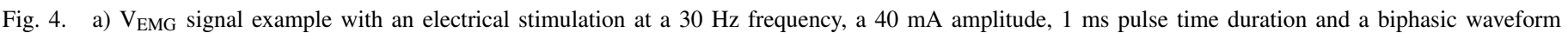

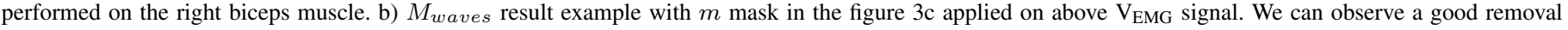
of artifact parts

\section{CONCLUSION}

In this study, a new stimulation artifact removal algorithm is presented. The technique, based on continuous wavelet transform, allows distinguishing the artifact parts and the M wave parts from an EMG signal. Then, once the artifact parts found, the treatment can suppress them. We performed tests for a lot of mother wavelets with good results excepted for "Sym3", "shan1-1", "gaus3" and "meyr" which let pass artifact parts or truncate $M$ wave parts. Instead of using standard mother wavelets, a new mother wavelet could be created by using artifact signal itself. In this way, the artifact removal could be adapted for any kind of 


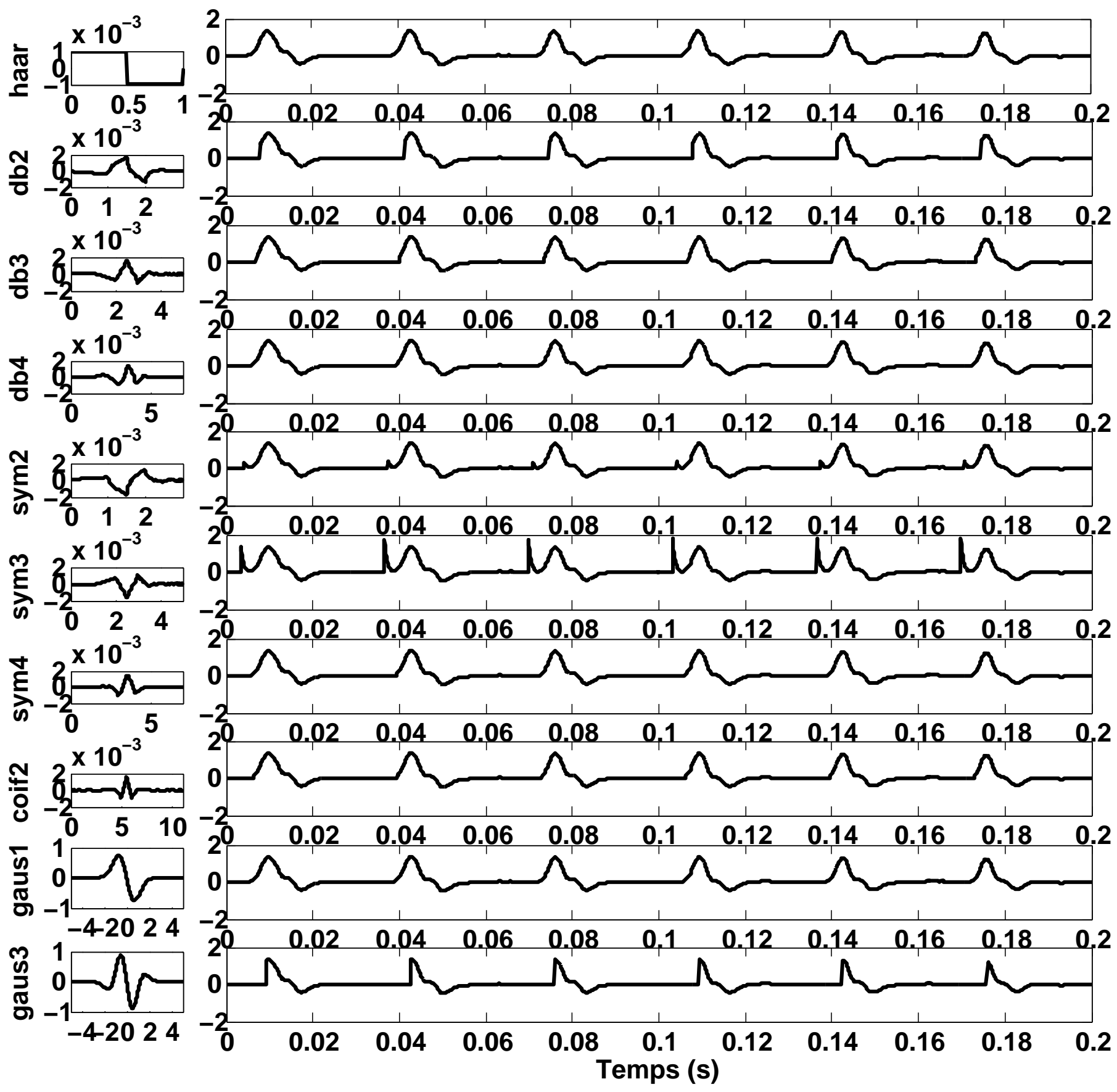

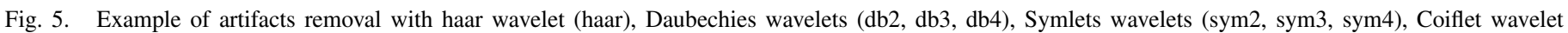
(coif2) and Gaussian wavelets (gaus1, gaus3) applied on the $\mathrm{V}_{\mathrm{EMG}}$ signal from the figure 2a.

stimulation pulses and to the muscle itself. This stimulation artifacts removal can be used as a signal pre-processing with an EMG signal during electrical stimulation contraction because that kind of signals contain both the electrical muscular activity and stimulation pulses artifacts. In a future work, we plan to compare the efficiency of each kind of wavelet in order to find the best one. However, our preliminary study shows good results with Haar wavelet. A comparison with existing artifacts removal process remains to be made to know possible differences with them. A possible limitation of our method is probably the execution time which is longer than threshold techniques because our method need to convert the EMG signal into wavelet domain.

REFERENCES

[1] M. Knaflitz and R. Merletti, "Suppression of stimulation artifacts from myoelectric-evoked potential recordings," Biomedical Engineering, IEEE Transactions on, vol. 35, no. 9, pp. 758-763, 1988. 


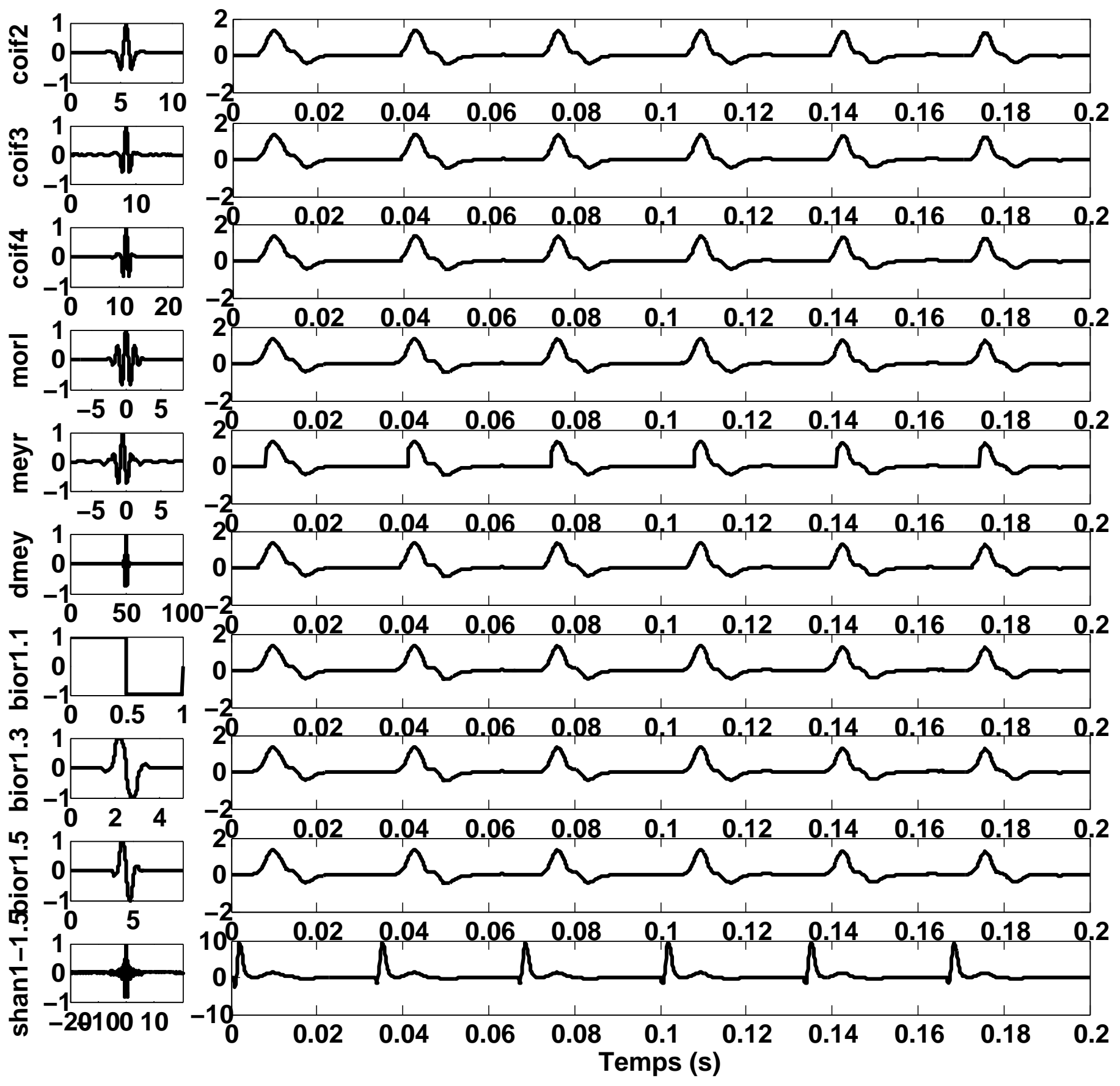

Fig. 6. Example of artifacts removal with Coiflet wavelet (coif2, coif3, coif3), Morlet wavelet (morl), Meyer wavelet (meyr, dmey), Biorthogonal wavelets (bior1.1, bior1.3, bior1.5,) and Shannon wavelet (shan1-1) applied on the $\mathrm{V}_{\mathrm{EMG}}$ signal from the figure 2a.

[2] M. Solomonow, R. Baratta, T. Miwa, H. Shoji, and R. D’Ambrosia, "A technique for recording the emg of electrically stimulated skeletal muscle.," Orthopedics, vol. 8, no. 4, p. 492, 1985.

[3] E. Roskar and A. Roskar, "Microcomputer based electromyographic recording system with stimulus artifact suppression," in Third Medical Conference on Biomedical Engineering, Portoroz Yugoslavia, 1983.

[4] R. Roby and E. Lettich, "A simplified circuit for stimulus artifact suppression," Electroencephalography and Clinical Neurophysiology, vol. 39, no. 1, pp. 85-87, 1975.

[5] J. Freeman, "An electronic stimulus artifact suppressor," Electroencephalography and clinical neurophysiology, vol. 31, no. 2, pp. 170-172, 1971.

[6] K. McGill, K. Cummins, L. Dorfman, B. Berlizot, K. Luetkemeyer, D. Nishimura, and B. Widrow, "On the nature and elimination of stimulus artifact in nerve signals evoked and recorded using surface electrodes," Biomedical Engineering, IEEE Transactions on, no. 2, pp. 129-137, 1982.

[7] D. O'Keeffe, G. Lyons, A. Donnelly, and C. Byrne, "Stimulus artifact removal using a software-based two-stage peak detection algorithm," Journal of neuroscience methods, vol. 109, no. 2, pp. 137-145, 2001.

[8] G. Harding, "A method for eliminating the stimulus artifact from digital recordings of the direct cortical response," Computers and biomedical research, 
vol. 24, no. 2, pp. 183-195, 1991.

[9] M. Yochum, T. Bakir, R. Lepers, and S. Binczak, "A real time electromyostimulator linked with emg analysis device," IRBM, vol. 34, no. 1, pp. 43-47, 2013.

[10] M. Yochum, T. Bakir, R. Lepers, and S. Binczak, "Estimation of muscular fatigue under electromyostimulation using cwt," IEEE transaction on Biomedical Engineering, vol. 59, no. 12, pp. 3372 - 3378, 2012.

[11] biodex, "http://www.biodex.com/physical-medicine/products/ dynamometers." Biodex system 3 pro.

[12] J. Serra, Image analysis and mathematical morphology. London.: Academic Press.[Review by Fensen, EB in: J. Microsc. 131 (1983) 258.] Cell size, Staining Microscopy Technique, Mathematics, General article Review article (PMBD, 185707888), 1982. 